\title{
Functional reconstitution of the insect odorant receptor co-receptor in a tethered lipid bilayer membrane
}

David Kleinheinz ${ }^{1}$, Chiara D’Onofrio ${ }^{1}$, Colm Carraher ${ }^{2}$, Ulrich Ramach ${ }^{3,4}$, Bernhard Schuster ${ }^{5}$, Wolfgang Knoll $^{1,6}$ and Jakob Andersson ${ }^{1, *}$

${ }^{1}$ Austrian Institute of Technology GmbH, Giefinggasse 4, 1210 Vienna, Austria

${ }^{2}$ The New Zealand Institute for Plant and Food Research, Auckland, New Zealand

${ }^{3}$ Technische Universität Wien, Wiedner Hauptstr. 8-10/134, 1040 Wien, Austria

${ }^{4}$ CEST Kompetenzzentrum für Oberflächentechnologie, Viktor Kaplan-Straße 2, 2700 Wiener Neustadt, Austria

${ }^{5}$ University of Natural Resources and Life Sciences (BOKU), Department of NanoBiotechnology, Institute for Synthetic Bioarchitectures, Muthgasse 11, 1190 Vienna, Austria

${ }^{6}$ Danube Private University, Steiner Landstraße 124, 3500 Krems an der Donau

* corresponding author

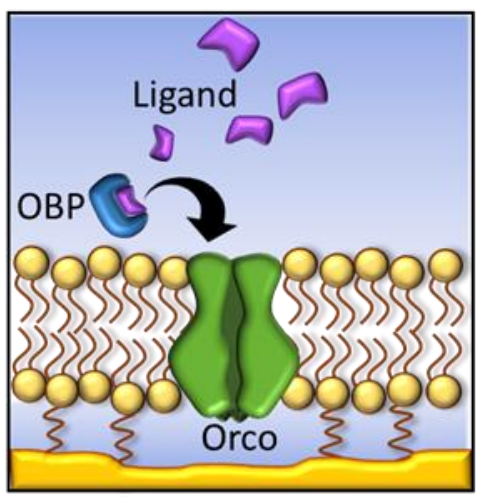

A tethered membrane architecture with expanded sub-membrane reservoir allows the functional reconstitution of the insect odorant receptor coreceptor (Orco). The membrane remains intact for more than 48 hours, allowing systematic examination of protein function with tools that cannot be applied to whole cells including electrochemical, optical and surface probe-based techniques.

Keywords: Membranes, Membrane proteins, ion channels, electrochemistry

\section{Contents}

Introduction

Results and discussion.

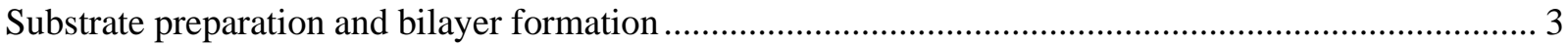

Verification of Orco function in a freestanding DPhyPC-bilayer ..................................................... 6

Verification of Orco function in the tethered membrane .................................................................. 7

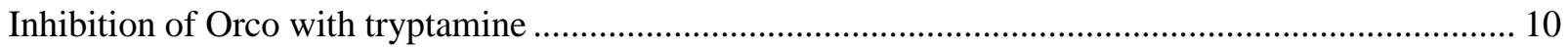

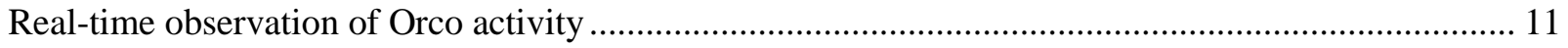

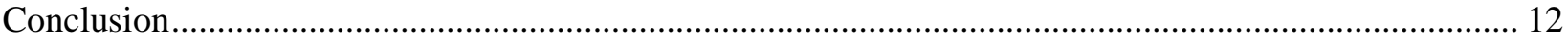

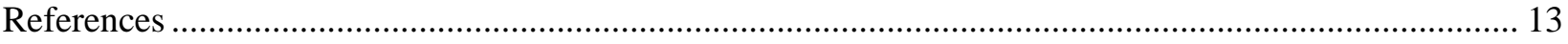




\section{Abstract}

As membrane proteins are among the most important drug targets, it is critical to study membrane proteins to improve drug design. However, due to the myriad roles fulfilled by the cellular membrane, it is a highly complex environment and challenging to study. Tethered membranes reproduce the basic physicochemical properties of the cellular membrane without their inherent complexity, and their high electrical resistance and stability makes them ideal to study membrane proteins, particularly ion channels. However, due to the close proximity of the membrane to the support and the reduced fluidity and high packing density, they are unsuitable to study larger membrane proteins. We present here a tethered membrane system into which the functional the odorant receptor coreceptor from Drosophila melanogaster, a tetrameric ionotropic receptor was incorporated and its sensitivity to various ligands was examined via electrochemical impedance spectroscopy and atomic force microscopy.

\section{Introduction}

Membrane proteins are among the most important biological structures for medicine, as around $60 \%$ of drugs have a target residing on the cell surface, for example GPCRs or ion channels. ${ }^{1}$ A better understanding of membrane proteins and the biological processes they are involved in would allow the development of more effective, more targeted drugs with fewer side effects and novel diagnostic methods using membrane protein-based biosensors. However, due to its importance, the cellular membrane is a highly heterogeneous and complex environment. ${ }^{2}$ Therefore, experiments conducted on live cells allow only very limited control over experimental parameters such as membrane composition, and techniques such as patch clamping are not well-suited for large-scale studies or experiments that require several hours to complete. Moreover, these systems allow only the use of techniques such as patch-clamping and fluorescence-based methods. While AFM can be applied to live cells, it is difficult to locate the protein of interest and there is significant interference from other membrane proteins and components.

Interference from other membrane components can be avoided by using model systems that replicate the physicochemical properties of the cellular membrane without its inherent complexity. They include for example free-standing lipid membranes and solid-supported lipid bilayers. However, free-standing membranes typically have very low stability ${ }^{3}$, their mechanical and long-term stability are often low, and they severely restrict the number of analytical tools that can be applied. Placing the membrane on a solid support increases stability and allows the use of tools such as surface plasmon resonance, atomic force microscopy (AFM) and more exotic techniques such as neutron scattering which thanks to recent advances in data processing even allows the elucidation of membrane protein structure ${ }^{4-6}$. However, the close proximity of the membrane to its support restricts the types of proteins that can be incorporated, as there is little space available to accommodate the sub-membrane domain of embedded proteins. Increasing the distance between membrane and spacer can remedy this, but at the cost of significantly reduced electrical resistance, making them unsuitable for ion channel studies. ${ }^{7}$ Resistance can be improved by covalently tethering the membrane to a solid support, increasing membrane lifespan to several months ${ }^{8}$ However, the membrane remains in very close proximity to the supporting material which, combined with the very dense packing of the inner leaflet, severely restricts their utility for the incorporation of membrane proteins. ${ }^{9}$ As a result, tethered membranes are best suited for the incorporation of pores or proteins that do not have significant sub-membrane domains and need not undergo conformational changes to open such as alpha hemolysin ${ }^{10}$ and other peptides ${ }^{11}$, gramicidin or boron-pump Bot $1^{12}$, an ortholog of BOR $1 .{ }^{13}$

The tethered membrane architecture presented here addresses these challenges, allowing the functional reconstitution of the odorant receptor coreceptor from Drosophila melanogaster (Orco), a tetrameric ligandgated ion channel. ${ }^{14,15}$ Orco is a part of the insect odorant receptor complex, a heteromultimer comprised of an individual olfactory receptor (OR), providing the specificity, and an olfactory receptor co-receptor (Orco) 
comprised of four subunits that act as an ion channel ${ }^{15}$ transporting cations such as $\mathrm{Na}^{+}$and $\mathrm{K}^{+}$and $\mathrm{Ca}^{2+}$. The sequence of ORs varies extensively, ${ }^{15}$ but sequence of Orco on the other hand is broadly conserved across species ${ }^{14}$ and fulfils several functions including transport of the receptor complex to the cell membrane after synthesis and maintenance of the structural integrity of the receptor in the membrane. ${ }^{5}$ The structure of Orco from Apocrypta bakeri has recently been solved ${ }^{14}$, but the overall structure of the OROrco complex has not yet been solved. While Orco can act as a ligand-gated ion channel by itself, it only responds to synthetic ligands such as VUAA1 which was first produced by Jones and colleagues. ${ }^{14,16}$ Although the structure of OR is unknown, significant similarities between the structure of OR and Orco subunits have been predicted based on their sequences. ${ }^{17}$

While the general mechanism of odorant sensing in insects has been elucidated ${ }^{15}$, much remains unknown such as the structure of the odorant receptor-co-receptor complex, the impact of the odorant receptor on ligand sensitivity and opening and closing staticstics and whether odorant binding proteins (OBPs) act only as a shuttle or play a more complex role in odorant detection. There is also some evidence that the Or-Orco complex can sense calcium levels in the presence of calmodulin, which may also modulate Orco sensitivity. ${ }^{15}$ It is critical to better understand insect olfaction as it is a central process in the insect life cycle. Knockout-studies showed that in the absence of Orco, ant behaviour is severely disrupted due to interference with airborne chemical communication. ${ }^{18,19}$ Mutations in the Orco gene have been shown to cause abnormal development and behaviour in ants. ${ }^{18,19}$ Interference in insect olfactory signalling pesticide use ${ }^{20}$ and pollution $^{21}$ is therefore a likely contributor to the loss of insect biomass observed globally ${ }^{22}$, and developing sustainable low-impact pesticides, for example based on pheromonal interference, could aid in the development of sustainable agricultural practices ${ }^{23,24}$, but this is not possible without a significantly better understanding of insect olfaction.

Until recently, it was technically challenging to express insect odorant receptors in a functional form and it was therefore not feasible to carry out studies on insect olfaction in model systems. However, insect odorant receptors have now been expressed by several groups, for example Carraher and colleagues ${ }^{25}$ and others ${ }^{26-}$ ${ }^{28}$. Orco was reconstituted into a tethered bilayer lipid membrane (tBLM) with an inner leaflet is based on 2,3-di-o-phytanyl-sn-glycerol-1-tetraethylene glycol-d,1-alpha-lipoic acid ester (DPhyTL) SAM on gold ${ }^{29}$ and the outer leaflet can be formed by any phospholipid via a variety of methods including solvent assisted bilayer formation, vesicle fusion or Langmuir-Blodgett transfer (see Figure 1b). These tBLM systems can retain their structure for several months ${ }^{8}$ and resistances in the G $\Omega$-range have been achieved ${ }^{30}$. They are therefore excellent platforms to study the function of ion channels as they are largely defect-free, permitting very little uncontrolled background current leakage. As there is no established model membrane system for the study of Orco, we focused on confirming the formation of a model system that replicates previously reported Orco behaviour.

\section{Results and discussion}

\section{Substrate preparation and bilayer formation}

To obtain tBLMs with sufficiently low defect density to study ion channels, substrates with very low roughness are required that are typically prepared via the template-stripping process described in more detail elsewhere. ${ }^{31}$ Briefly, gold is deposited on a polished silicon substrate via physical vapor deposition and a glass slide is applied to the exposed gold surface via an epoxide-based glue. The silicon support is removed immediately prior to use, exposing the ultra-flat gold surface previously in contact with the silicon (see Figure 1a for a schematic overview). The high electrical resistance of DPhyTL-based tBLMs is in part due to the excellent packing of the inner leaflet. However, the high packing density of DPhyTL also restricts the sub-membrane space to around 1-2 nm and a low water content of a round 5\% water. ${ }^{9}$ This architecture does 
therefore not allow the incorporation of larger membrane proteins, particularly those with extensive submembrane domains. Sub-membrane hydration can be improved to $25 \%$ through a reduction of the tethering density by diluting the inner leaflet by various means, creating sparsely tethered bilayer lipid membranes (stBLMs, see Figure 1C), ${ }^{32}$ but we found that this architecture did not allow for the formation of stable lipid membranes containing Orco. Structural analysis of Orco has shown that its sub-membrane domain extends around 3-4 nm below the membrane surface. ${ }^{14}$ Thus, the typical amount of space available under a tBLM is insufficient to incorporate Orco, preventing the formation of a lipid bilayer. Bilayer formation therefore required an increase in sub-membrane space. This can be achieved by increasing the length of the tether beyond the four ethylene oxide (EO) units of DPhyTL. However, even small increases of tether length to six EO units reduce the maximum achievable membrane resistance by an order of magnitude or more ${ }^{9}$, making them unsuitable for ion channel studies. The amount of available sub-membrane space could also be increased by using a substrate with increased roughness that effectively provides cavities with additional sub-membrane space to accommodate proteins (see Figure 1D). Preparing substrates via thermal evaporation of gold yielded substrates with an RMS roughness of 3-5 nm (see Figure 3A). On these, bilayers either could not form at all, or rapidly lost stability after formation. We therefore replaced the silicon substrate in the template stripping process by one with slightly higher surface roughness, obtaining substrates with an RMS roughness of $1.65 \pm 0.22 \mathrm{~nm}$, to make rough template-stripped gold (rTSG, see Figure 3B). The rTSG substrate has a peak-to-valley distance of up to $8 \mathrm{~nm}$, which can easily accommodate the sub-membrane domain of Orco. The substrate was functionalized with a sparsely tethered inner leaflet formed by immersion in an ethanolic solution of DPhyTL and mercaptoethanol with a combined concentration of $5 \mathrm{mM}$ at $8^{\circ} \mathrm{C}$ for 18 hours. We tested anchorlipid/spacer mixtures of 4:1, 1:1, 1:2 and 1:4. Stable bilayers formed only at a tether/spacer ratio of 1:4. The outer leaflet of the membrane was assembled by fusion of $70 \mathrm{~nm}$ vesicles (for DLS data see supporting information Figure S 11).comprised of DPhyPC and Orco protein (see Figure 1D) over 18 hours at $30^{\circ} \mathrm{C}$.
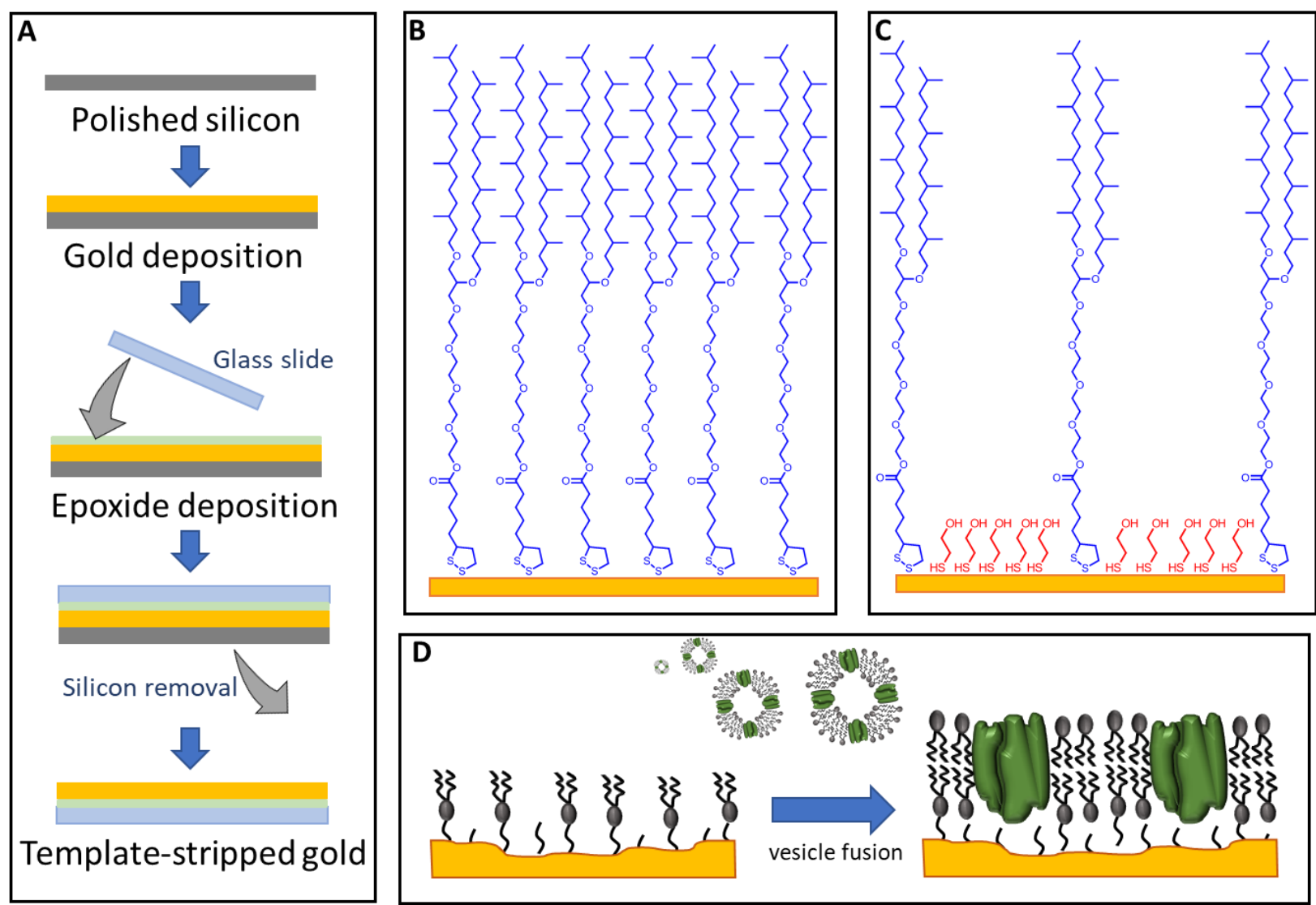
Figure 1: A) substrate preparation via template-stripping. B) Chemical structure of a tBLM comprised of DPhyTL (blue). C) Chemical structure of an stBLM where DPhyTL is diluted with mercaptoethanol (red), affording additional sub-membrane space to accommodate membrane proteins. D) Bilayer formation via vesicle fusion to a SAM comprised of DPhyTL and mercaptoethanol. E) $s t B L M$ on a flat substrate where embedded proteins may come into contact with the substrate. F) stBLM on substrate with increased roughness to accommodate embedded proteins.

Table 1 shows an overview of the roughness produced by various methods of surface preparation. Rough template-stripped gold substrates allowed the formation of stable tethered membranes, which was indicated by a decrease in surface roughness from 1.65 to $0.38 \mathrm{~nm}$ (see Figure $3 \mathrm{c}$ and Table 1). Figure $\mathrm{S} 3$ shows an AFM scan of a membrane patch containing a pinhole defect with a depth of around $5 \mathrm{~nm}$ which corresponds to the approximate thickness of a tethered lipid bilayer. ${ }^{33}$

A

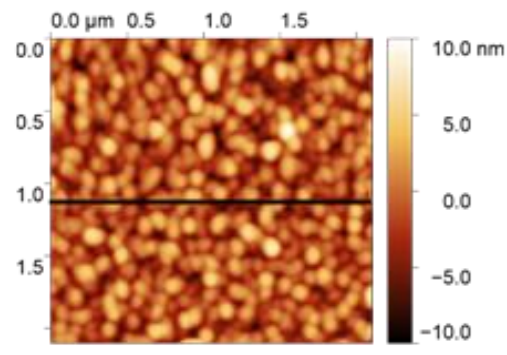

B

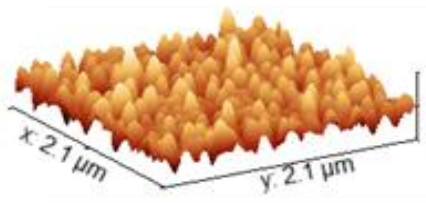

C

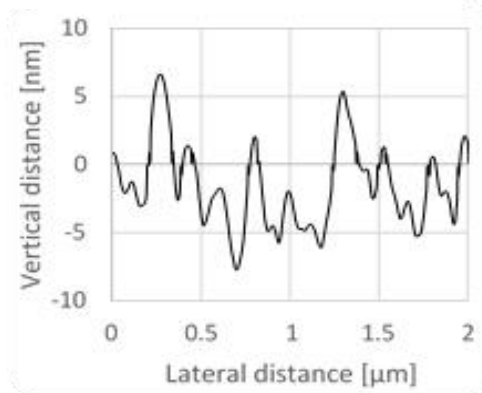

D

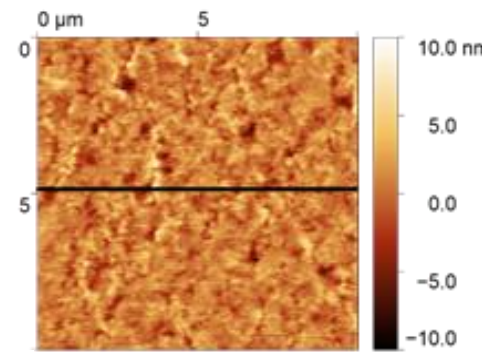

$\mathrm{E}$

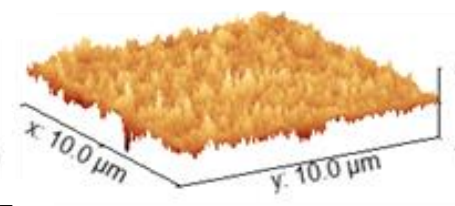

$\mathbf{F}$

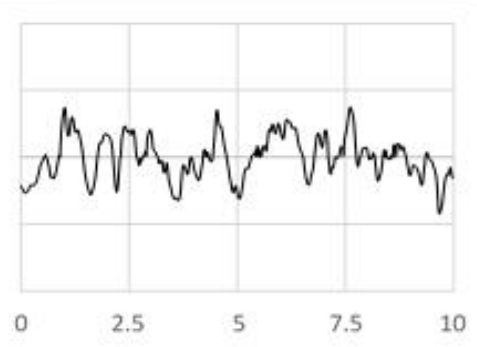

G

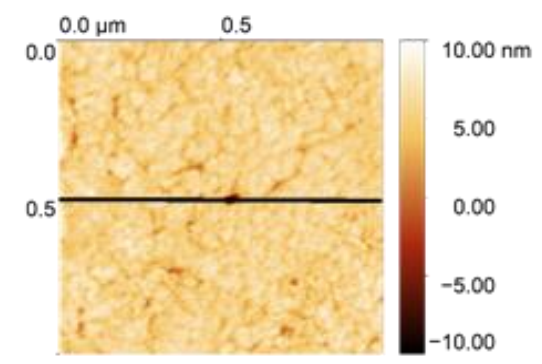

H

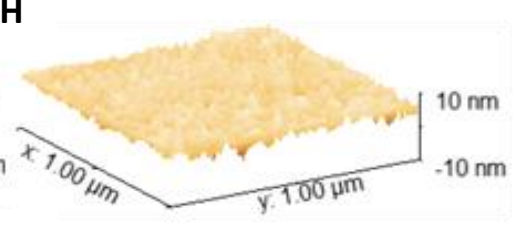

I

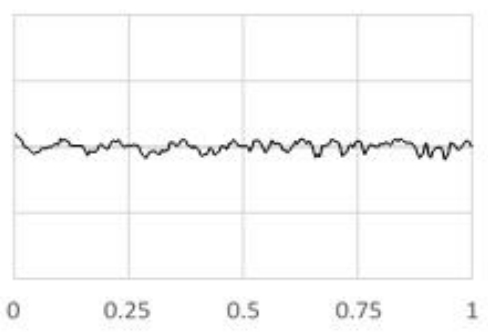

Figure 2: AFM characterization of evaporated gold (A-C) compared to rough TSG $(D-F)$ and a lipid bilayer on rough TSG $(G-I)$.

The RMS roughness of the Orco-functionalised stBLM was around $0.1 \mathrm{~nm}$ higher than that of an unfunctionalized tBLM. ${ }^{34}$ This might be partially attributed to the rougher support and/or the embedded proteins, although Orco does not have any features that significantly extend above the membrane ${ }^{14}$, making it difficult to detect the protein with AFM among the natural roughness of a tBLM.

Table 1: Surface roughness of various substrates before and after lipid bilayer formation

\begin{tabular}{|c|c|c|}
\hline & Substrate & Bilayer \\
\hline Thermally evaporated gold & $3-5 \mathrm{~nm}$ & Does not form \\
\hline Rough TSG & $1.65 \pm 0.22 \mathrm{~nm}$ & $0.377 \pm 0.07 \mathrm{~nm}$ \\
\hline
\end{tabular}




\begin{tabular}{|l|l|l|}
\hline Smooth TSG & $0.375 \pm 0.13 \mathrm{~nm}$ & 0.27 (see ref. $^{30}$ ) \\
\hline
\end{tabular}

The roughness of an Orco-functionalised stBLM could not be compared to a bilayer comprised only of phospholipids on an rTSG substrate, as protein-free lipid bilayers with comparable electrical resistance and stability cannot form in the absence of the protein by either vesicle fusion or solvent-assisted bilayer formation on these substrates. It appears that that the presence of proteins is required to enable the formation of stable lipid bilayers. Electrochemical impedance spectroscopy (EIS) confirmed the formation of lowdefect lipid bilayers, with resistances in the range of 7-20 M $\Omega \mathrm{cm}^{2}$ which is in good agreement with other tethered membrane systems. ${ }^{18}$ Unlike tBLMs comprised only of phospholipids, Orco-tBLMs had capacitance of the membrane systems was around 10-20 $\mu \mathrm{F} \mathrm{cm}^{-2}$, which is an order of magnitude higher than the values of $0.5-1 \mu \mathrm{F} \mathrm{cm}{ }^{-2}$ typically reported for tethered membrane systems. ${ }^{9}$ The increased capacitance can be attributed to the presence of membrane proteins which contribute additional surface charges and increased roughness (and therefore surface area which increases absolute capacitance) to the membrane. The membranes retained their stability over the period of at least $24 \mathrm{~h}$, which significantly exceeds the duration of a typical experiment of around 2 hours. (see Table 2 and Figure S 5 in the supplementary information for Bode plots).

Table 2: Stability of an Orco-functionalized stBLM over time

\begin{tabular}{|c|c|c|}
\hline & $\begin{array}{c}\text { Resistance (M } \Omega \\
\left.\mathbf{c m}^{2}\right)\end{array}$ & $\begin{array}{c}\text { Capacitance }(\mu \mathrm{F} \\
\left.\mathrm{cm}^{-2}\right)\end{array}$ \\
\hline $0 \mathrm{~h}$ & $13.4 \pm 2.4$ & $10.1 \pm 0.4$ \\
\hline $2 \mathrm{~h}$ & $12.5 \pm 2.1$ & $10.2 \pm 0.4$ \\
\hline $24 \mathrm{~h}$ & $13.2 \pm 1.7$ & $9.8 \pm 0.3$ \\
\hline
\end{tabular}

\section{Verification of Orco function in a freestanding DPhyPC-bilayer}

To confirm that Orco functionality in membranes comprised only of DPhyPC was not altered, we incorporated the ion channel in free-standing bilayer lipid membranes formed via the tip-dip approach ${ }^{35}$. After membrane formation had been confirmed, Orco was introduced via the addition of liposomes from which it incorporates spontaneously into the free-standing membrane. According to the electrophysiological characterization of Orco by Butterwick and colleagues, the maximum response of Orco was produced at a concentration of $100 \mu \mathrm{M}$ VUAA $1 .{ }^{14}$ In the free-standing membranes, current spikes with a magnitude of 2 pA appear that are indicative of single channel activity, which is in excellent in agreement with previous reports ${ }^{16}$ (see Figure 3). Additional recordings of Orco ion channels embedded in BLMs are shown in Figure $\mathrm{S} 10$ in the supporting information. 

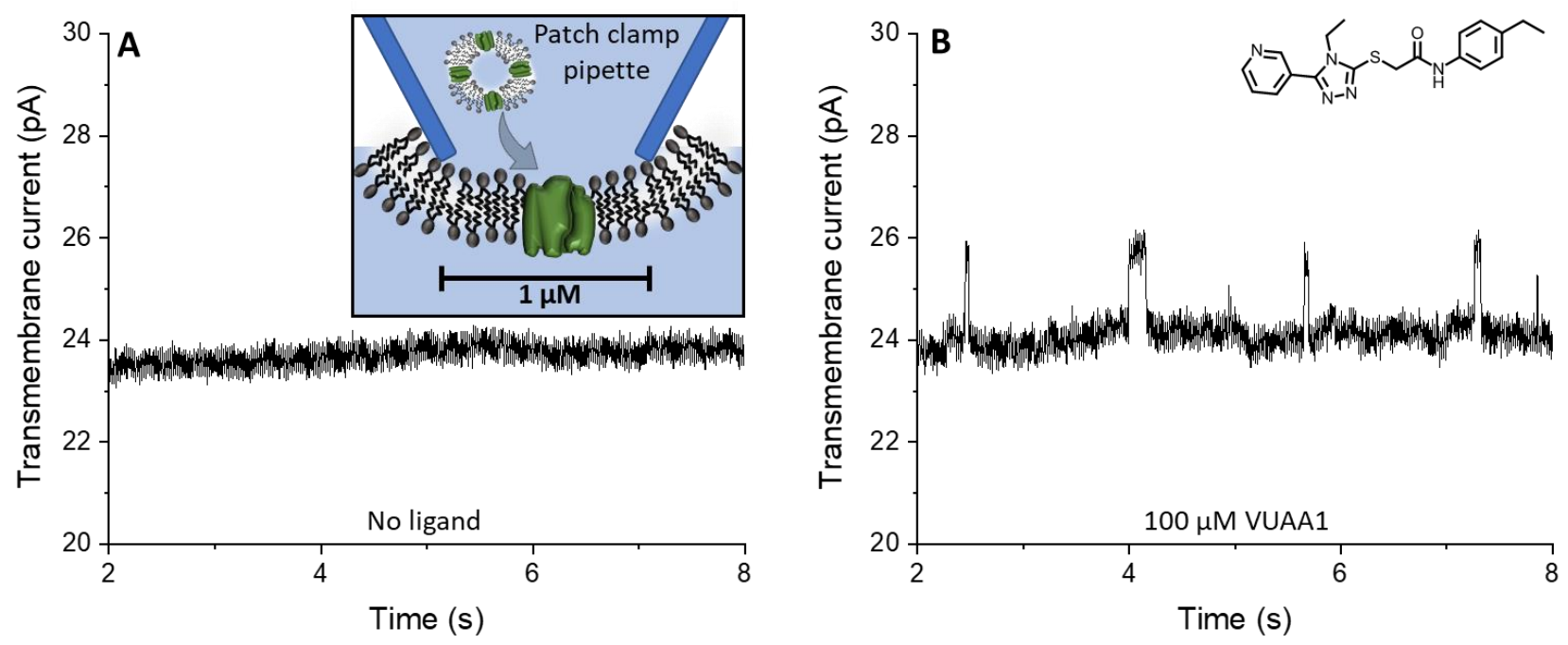

Figure 3: Single-channel activity of an Orco-functionalized BLM before ligand addition (A) and after addition of $100 \mu \mathrm{M}$ VUAA1 (B).

Verification of Orco function in the tethered membrane

Having confirmed the normal function of Orco reconstituted in DpyhPC membranes from vesicles, we examined the functionality of the embedded proteins. VUAA1 was added to the pre-formed bilayers, which should cause a reversible reduction of membrane resistance upon opening of the embedded ion channels. As VUAA1 has poor water solubility, it is typically solubilized by adding $0.5-1$ vol-\% DMSO to the buffer solution. ${ }^{14}$ No significant changes in membrane topology could be seen upon addition of VUAA1 in AFM, therefore ion channel function could only be observed electrically. Orco opening in the presence of VUAA1 was reproduced in the tethered membrane systems shown here (see Table 3), where an incubation of the bilayer with $100 \mu \mathrm{M}$ VUAA1 dissolved in a $138 \mathrm{mM}$ PBS solution containing 1 vol-\% DMSO caused a reversible $30 \%$ reduction in membrane resistance after two hours and a reduction of $80 \%$ after 18 hours.

While this confirmed the functionality of Orco, the response was much slower than would be the case in free-standing membranes. As there are no other reports of Orco in planar membranes against which these data could be benchmarked, it cannot be confirmed if this is typical behaviour for Orco in tethered membrane systems. We therefore explored the use of odorant binding proteins to deliver VUAA1 to the receptor instead, as they are optimized to transfer hydrophobic molecules across an aqueous reservoir and unlike organic solvents do not pose a risk of damaging the membrane (see supplementary information Table S 2). Among the OBPs available to us, we selected EcorOBP15-m1, a mutant of EcorOBP15 of the hoverfly Eupeodes corollae ${ }^{36}$ in which Phe61 was replaced by Leu, as it showed the highest affinity for VUAA1 in competitive binding experiments (see Figure S2 in the supporting information) with an estimated $\mathrm{K}_{\mathrm{D}}$ of 10 $\mu \mathrm{M}$. As control, we chose AmelOBP14 from Apis mellifera, which has a high affinity for Eugenol and no appreciable affinity for VUAA $1 .{ }^{26}$ To introduce VUAA1 using odorant binding proteins instead of DMSO, a VUAA1 stock solution was prepared in ethanol (maximum final ethanol concentration of $0.3 \%$ in PBS solution) and then added to a $138 \mathrm{mM}$ PBS solution containing $2 \mu \mathrm{M}$ OBP with which it was incubated for 30 minutes prior to addition to the membrane.

Table 3 and Figure 4 show the effectiveness of solubilizing VUAA1 with DMSO, AmelOBP14 and EcorOBP15-m1. Introduction of VUAA1 via AmelOBP14 was less effective than DMSO, achieving a reduction in membrane resistance of $50 \%$ after 18 hours. The reduction in membrane resistance when VUAA1 was added with AmelOBP14 (even though it had no appreciable affinity for VUAA1) suggests that some small amount of the ligand could still bind to the OBP. 
When the ligand was introduced with EcorOBP15-m1, membrane resistance was reduced by $80 \%$ after 30 minutes and more than $95 \%$ after two hours. In all cases, ion channel opening was reversible as the initial membrane resistance could be restored by rinsing the bilayers with 5 cell volumes of $138 \mathrm{mM}$ PBS solution.

Table 3: Ion channel response of Orco to various methods of solubilizing VUAA1

\begin{tabular}{|c|c|c|}
\hline & Resistance $\left(\mathrm{M} \Omega \mathrm{cm}^{2}\right)$ & Capacitance $\left(\mu \mathrm{F} \mathrm{cm}^{-2}\right)$ \\
\hline \multicolumn{3}{|c|}{$100 \mu \mathrm{M}$ VUAA1, $138 \mathrm{mM}$ PBS + 1\% DMSO } \\
\hline Initial bilayer & $18.2 \pm 1,2$ & $10.3 \pm 0.03$ \\
\hline $2 \mathrm{~h}$ & $13.8 \pm 0.8$ & $10.5 \pm 0.01$ \\
\hline $18 \mathrm{~h}$ & $3.97 \pm 0.2$ & $10.8 \pm 0.02$ \\
\hline PBS rinse & $16.1 \pm 0.4$ & $10.1 \pm 0.05$ \\
\hline \multicolumn{3}{|c|}{$1 \mu \mathrm{M}$ VUAA1, $2 \mu \mathrm{M}$ OBP } \\
\hline Initial bilayer & $12.5 \pm 2.7$ & $8.9 \pm 0.13$ \\
\hline AmelOBP14, 2h & No change & No change \\
\hline AmelOBP14, $18 \mathrm{~h}$ & $6.8 \pm 2.1(-46 \%)$ & $9.6 \pm 0.20$ \\
\hline Initial Bilayer & $10.5 \pm 2.3$ & $9.6 \pm 0.17$ \\
\hline EcorOBP15-m1, $2 \mathrm{~h}$ & $2.6 \pm 0.2,(-75 \%)$ & $15.7 \pm 0.16,(+61 \%)$ \\
\hline EcorOBP15-m1, $18 \mathrm{~h}$ & No further change & No further change \\
\hline PBS rinse & $19.1 \pm 8.6$ & $9.5 \pm 0.16$ \\
\hline \multicolumn{3}{|c|}{$100 \mu \mathrm{M}$ VUAA1, $2 \mu \mathrm{M}$ EcorOBP15-m1 } \\
\hline Initial bilayer & $2.03 \pm 0.19$ & $13.0 \pm 0.3$ \\
\hline $30 \mathrm{~min}$ & $0.41 \pm 0.02,(\mathbf{- 8 0 \%})$ & $19.3 \pm 0.4 \quad(+\mathbf{4 8 \%})$ \\
\hline $2 \mathrm{~h}$ & $0.07 \pm 0.03,(-97 \%)$ & $31.7 \pm 0.1(+244 \%)$ \\
\hline PBS rinse & $1.18 \pm 0.31$ & No change \\
\hline
\end{tabular}

In addition to changes in membrane resistance, membrane capacitance increased by around $50 \%$ after two hours in the presence of $1 \mu \mathrm{M}$ VUAA1 and roughly doubled in the presence of $100 \mu \mathrm{M}$ VUAA1, indicating that the sub-membrane reservoir became populated by ions upon opening of the ion channel, which has been reported previously when ion transport of the membrane occurs either through transporters or defects. ${ }^{10,37}$ As the capacitance remained high even after membrane resistance was restored when the ligand was removed suggests that the capacitance increase cannot be attributed to additional surface charges that might become exposed upon ion channel opening. 

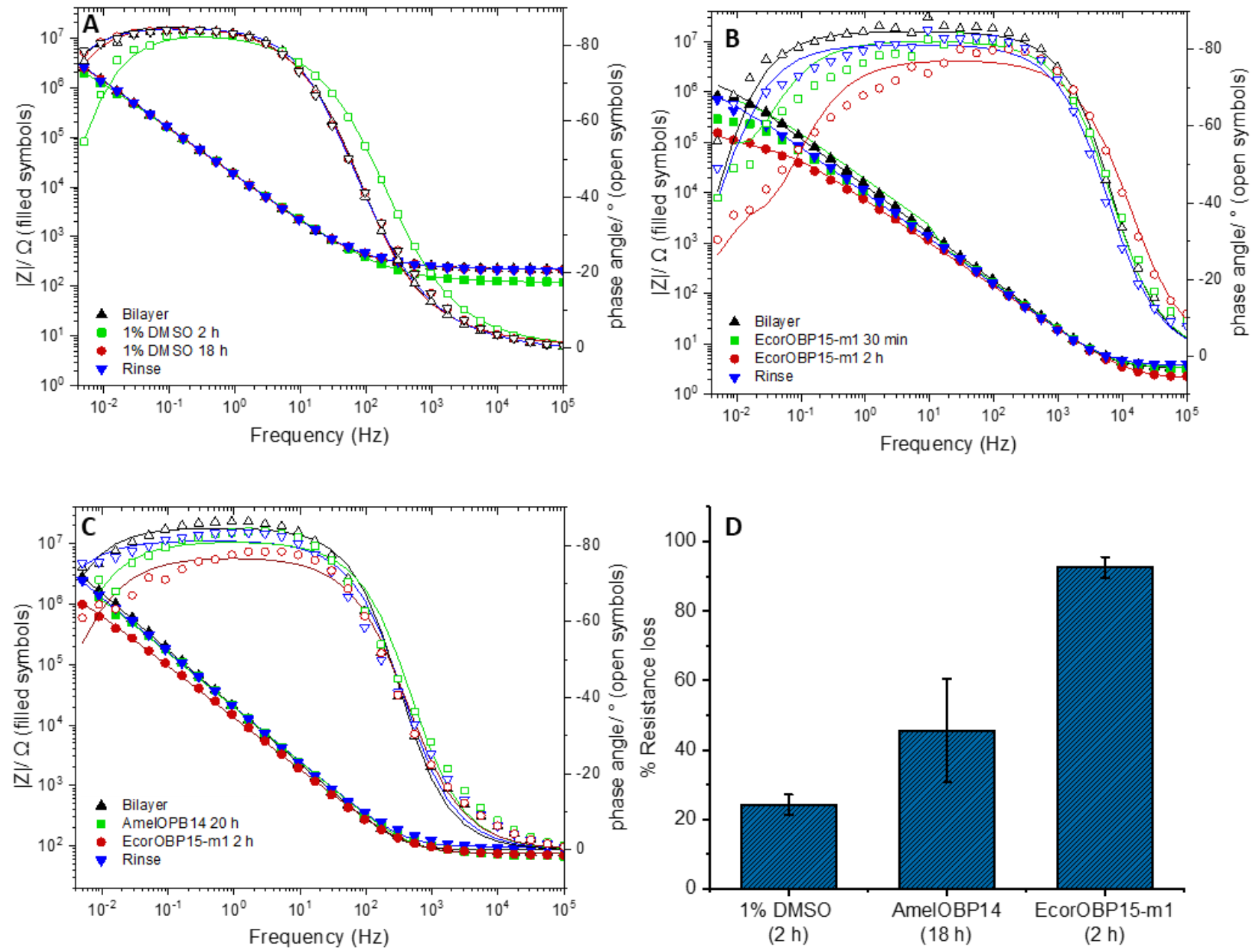

Figure 4: Bode plots of Orco-tBLMs after different methods of introducing $100 \mu M$ VUAA1. A) $1 \%$ DMSO in PBS, B) $2 \mu M$ OBP 15-m1, C) Comparison of AmelOBP14 and EcorOBP15-m1, D) relative reduction in membrane incubation of $100 \mu M$ VUAA1 with different solubilizing agents

As there was no further reduction in membrane resistance after two hours of incubation with $100 \mu \mathrm{M}$ VUAA1 introduced by EcorOBP15-m1, we assume that all ion channels inserted in the correct orientation and their native structure have opened. The EIS data was fitted to a slightly altered equivalent circuit in which the bilayer resistance is fitted with two parallel resistors, one of which represents the membrane resistance and is fixed at the value fitted prior to ligand addition and the second represents the ion channel resistance and is allowed to vary (see Supporting information, Figure S 4, circuit 4 for a schematic of the equivalent circuit). The membrane resistance was kept at $2.03 \mathrm{M} \Omega \mathrm{cm}^{2}$ (the initial membrane resistance, shown in Table 3), which resulted in a fitted combined resistance of all open ion channels of $72 \mathrm{k} \Omega$. Using the previously reported single-channel currents of $2 \mathrm{pA}$ at $-80 \mathrm{mV},{ }^{14}$ we obtain a conductivity of $3.3 \times 10^{-11}$ $\mathrm{S}$. The reported diameter of the Orco pore is $3 \mathrm{~nm},{ }^{14}$ which yields a normalised resistance of $0.085 \Omega \mathrm{cm}^{2}$ per pore. By combining this knowledge with the fitted resistance of all combined Orco proteins of $72 \mathrm{k} \Omega$ $\mathrm{cm}^{2}$, we can estimate that the membrane contains $2.55 \pm 1.08 \times 10^{7}$ ion channel $/ \mathrm{cm}^{2}$ (see supporting information for full calculation). All bilayers were formed on membranes with an area of $0.28 \mathrm{~cm}^{2}$.

To determine the impact of VUAA1 concentration on ion channel opening in the presence of EcorOBP15$\mathrm{m} 1$, we exposed the membrane to increasing concentrations of the ligand while keeping the OBP concentration at $2 \mu \mathrm{M}$. An overview of the data can be seen in Table 4 (all parameters and Bode plots can be found in the supporting information, Table $S 2$ an Figure $S 6$ ). 
Table 4: Resistance decrease after $2 \mathrm{~h}$ incbuation of 1-100 $\mu \mathrm{M}$ VUAA1 with $2 \mu \mathrm{M}$ EcorOBP15-m1(for full data set see Table S 2). Errors are as reported by the fitting program.

\begin{tabular}{|c|c|}
\hline $\begin{array}{c}\text { VUAA1 } \\
\text { concentration }\end{array}$ & $\begin{array}{c}\text { Resistance decrease } \\
\text { after 30 min }\end{array}$ \\
\hline $1 \mu \mathrm{M}$ & $37 \pm 5 \%$ \\
\hline $10 \mu \mathrm{M}$ & $48 \pm 8 \%$ \\
\hline $100 \mu \mathrm{M}$ & $91 \pm 10 \%$ \\
\hline
\end{tabular}

While OBPs do not have any effect on membrane resistance and cannot trigger ion channel opening when they do not carry a ligand (see supporting information, Figure S 7 / Table S 3), we tested if other ligands not specific to Orco might have an effect when introduced via OBPs. Eugenol and were selected for this, as they have a high affinity for AmelOBP $14^{38}$ and EcorOBP15-m1, respectively but have no effect on Orco. Both ligands were introduced with the same concentration as VUAA1 $(100 \mu \mathrm{M})$. Neither eugenol nor geranyl acetate in any combination with OBPs had any effect on membrane resistance (see Table 5). The addition of eugenol in combination with DMSO resulted in a reduction of membrane resistance by $50 \%$ over a period of two hours, whereas eugenol had no effect on membrane resistance when introduced with AmelOBP14. As DMSO is often used to facilitate drug uptake in cells by increasing lipid bilayer permeability and lipid flip flop behaviour, ${ }^{39}$ the most likely cause of the reduction in membrane resistance is the formation a membrane-damaging complex between eugenol and DMSO.

Addition of eugenol with AmelOBP14 caused a 50\% increase in capacitance, which could indicate that eugenol interacts with a lipid bilayer. It is possible that the nonpolar region of eugenol inserts into the lipid membrane, causing changes in its dielectric properties or causing the formation of lesions without causing the formation of conductive defects such that only a change in membrane capacitance due to increased surface roughness occurs. Finally, in order to confirm the role of EcorOPB15-m1 as a transporter of VUAA1 to the Orco protein, we pre-incubated the OBP with geranyl acetate before adding VUAA1. In this combination, Orco activation was significantly reduced, with membrane resistance only changing from 1.17 $\mathrm{M} \Omega \mathrm{cm}^{2}$ to $0.88 \mathrm{M} \Omega \mathrm{cm}^{2}$ (see supplementary information, Table S 3), confirming the role of OBP15M1 as a transporter for VUAA1.

\section{Inhibition of Orco with tryptamine}

As described by Chen et al, tryptamine (2-(3-Indolyl)ethylamine) can competitively inhibit the function of the Orco-protein, resulting in minimal or no response upon contact with potential agonists. ${ }^{40}$ When the membrane was pre-incubated with tryptamine, no significant changes in membrane resistance occurred even after an incubation time of $48 \mathrm{~h}$. There was a $30 \%$ increase in membrane capacitance over this period of time, though, which could be attributed tryptamine binding to Orco and/or the membrane surface. When the system was rinsed and VUAA1 in combination with OBP15M1 was introduced, a small response to VUAA1 could be observed (see Figure S 8 and Table S 4), with resistance being reduced by around 50\% after two hours. Likely, some but not all ion channels recovered their functionality after removal of tryptamine and further rinsing might have recovered additional ion channel functionality. 
Table 5: Effect of various ligands for which Orco has no sensitivity introduced by OBP or organic solvents.

\begin{tabular}{|c|c|c|}
\hline & Resistance $\left(\mathrm{M} \Omega \mathrm{cm}^{2}\right)$ & Capacitance $\left(\mu \mathrm{F} \mathrm{cm}^{-2}\right)$ \\
\hline \multicolumn{3}{|c|}{$100 \mu \mathrm{M}$ Eugenol in DMSO } \\
\hline Initial Bilayer & $8.29 \pm 0.74$ & $11.1 \pm 0.02$ \\
\hline $1 \mathrm{~h}$ & $4.62 \pm 0.68(-45 \%)$ & $12.9 \pm 0.06(+\mathbf{1 4 \%})$ \\
\hline $2 \mathrm{~h}$ & $3.05 \pm 0.23(-63 \%)$ & $14.3 \pm 0.02(+29 \%)$ \\
\hline \multicolumn{3}{|c|}{$100 \mu \mathrm{M}$ Eugenol + AmelOBP14 } \\
\hline Initial Bilayer & $0.97 \pm 0.11$ & $19.90 \pm 0.48$ \\
\hline $1 \mathrm{~h}$ & $0.83 \pm 0.05$ & $29.25 \pm 0.37(+\mathbf{4 7 \%})$ \\
\hline $2 \mathrm{~h}$ & $1.05 \pm 0.07$ & $27.40 \pm 0.35$ \\
\hline \multicolumn{3}{|c|}{$100 \mu \mathrm{M}$ Geranyl acetate in DMSO } \\
\hline Initial Bilayer & $1.46 \pm 0.11$ & $24.58 \pm 0.32$ \\
\hline $1 \mathrm{~h}$ & $1.09 \pm 0.08$ & $24.58 \pm 0.37$ \\
\hline $2 \mathrm{~h}$ & $1.22 \pm 0.09$ & $24.61 \pm 0.38$ \\
\hline \multicolumn{3}{|c|}{$100 \mu \mathrm{M}$ Geranyl + EcorOBP15-m1 } \\
\hline Initial Bilayer & $1.48 \pm 0.11$ & $20.41 \pm 0.30$ \\
\hline $1 \mathrm{~h}$ & $1.18 \pm 0.11$ & $22.06 \pm 0.42$ \\
\hline $2 \mathrm{~h}$ & $1.22 \pm 0.48$ & $16.08 \pm 0.48$ \\
\hline \multicolumn{3}{|c|}{ Inhibition with $100 \mu \mathrm{M}$ tryptamine } \\
\hline Initial Bilayer & $1.17 \pm 0.16$ & $33.5 \pm 10.2$ \\
\hline $\begin{array}{l}100 \mu \mathrm{M} \text { VUAA1 } 1+2 \mu \mathrm{M} \\
\text { EcorOBP15-m1 } 1 \mathrm{~h}\end{array}$ & $0.74 \pm 0.11 \quad(-36 \%)$ & $43.5 \pm 11.7(+\mathbf{3 0 \%})$ \\
\hline $\begin{array}{l}100 \mu \mathrm{M} \text { VUAA } 1+2 \mu \mathrm{M} \\
\text { EcorOBP15-m1 } 48 \mathrm{~h}\end{array}$ & $0.88 \pm 0.19$ & $46.1 \pm 16.6$ \\
\hline PBS flush & $0.89 \pm 0.11$ & $38.1 \pm 6.3$ \\
\hline
\end{tabular}

The time required to reach maximum ion channel activity in EIS experiments is long compared to the time scale at which typical patch clamping experiments are carried out, which is in the range of seconds or milliseconds. Some of this delay can be attributed to the size of the reservoir across which the ligand must travel after addition (around $1 \mathrm{~mL}$ ), as it was introduced to the cell at some distance from the surface to avoid disturbing the membrane. Additionally, tethering reduces the fluidity of the membrane ${ }^{7}$ compared to free-standing and solid-supported membranes, possibly inhibiting Orco function. However, as the sensitivity of Orco embedded in tBLMs to VUAA1 as well as inhibition by tryptamine are in qualitative agreement with previous reports, this model system is clearly useful to gain a qualitative understanding of fundamental interactions. It is therefore feasible to perform initial screenings of large compound libraries against Orco reconstituted in robust model membrane systems before the observed behaviour is examined further via electrophysiology. However, to further test the utility of the model membrane system, we observed the ligand addition process to a tethered membrane in real time via chronoamperometry.

\section{Real-time observation of Orco activity}

To monitor ion channel activity in real time, we recorded the transmembrane current during ligand addition at a potential of $-80 \mathrm{mV}$. Prior to ligand addition, an initial background current of a few hundred $\mathrm{nA}$ was recorded as the membrane was charged by the applied potential. This quickly decreased to a base level of 1-2 $\mathrm{nA}$ as the membrane was charged by the applied potential of $-80 \mathrm{mV}$ which was chosen based on previously published ion channel behavior ${ }^{14}$. A few seconds after ligand addition, current spikes up to 10- 
fold above the background were recorded (Figure 5a), as some ion channels rapidly responded upon ligand addition. Recordings after the membrane was incubated with VUAA1 for 60 minutes showed a steady current around the level of the previously recorded peaks around $10 \mathrm{nA}$ (Figure 5b). Upon rinsing, the transmembrane current returned to the level recorded prior to ligand addition. Additional chronoamperometry measurements during ligand addition as well as control experiments introducing VUAA1 to a membrane without Orco are shown in Figure $S 9$ in the supporting information.
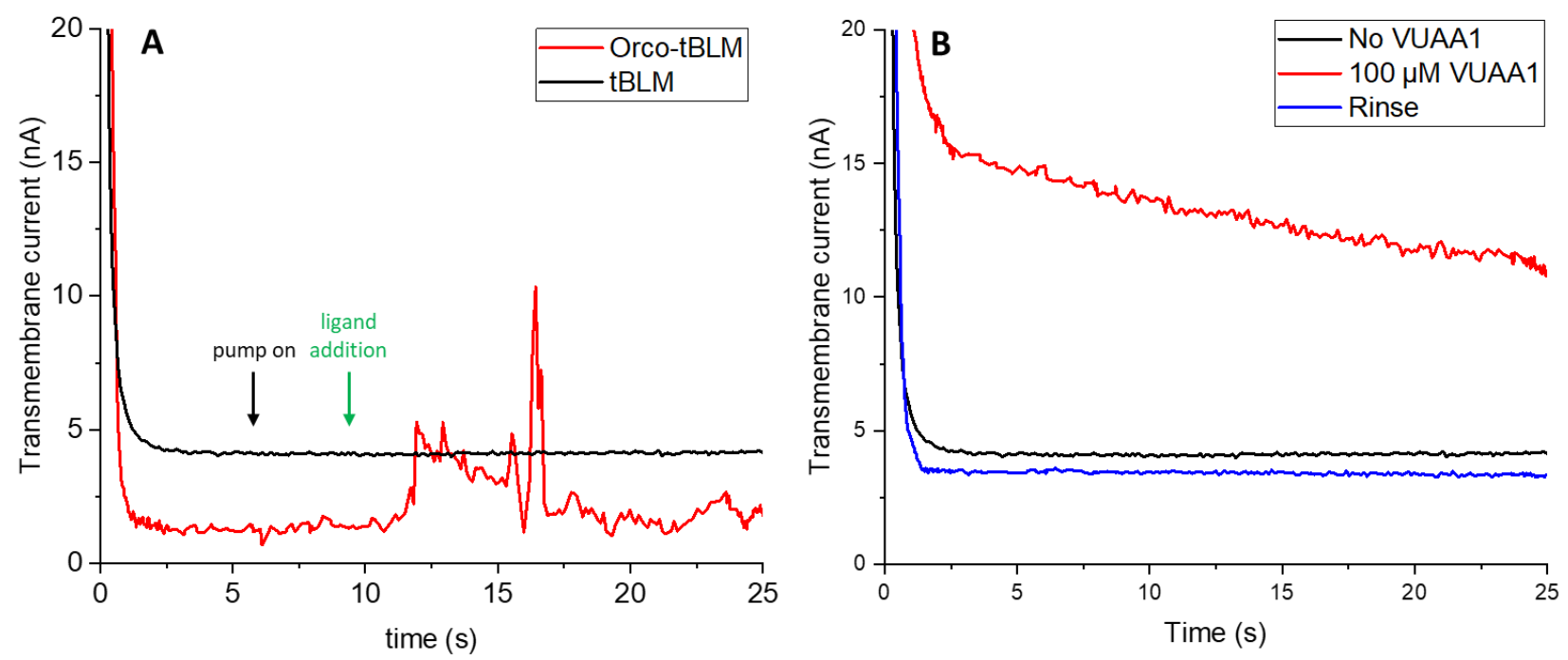

Figure 5: Real-time chronoamperometric observation of VUAA1 activity during ligand addition (A) and 60 minutes after ligand addition (B). All recordings were made at a potential of $80 \mathrm{mV}$.

In the configuration used here, it was not possible to record the activity of single ion channels as this would require background currents in the low pA-range that can only be reached when membrane resistance in in the G $\Omega$-range. However, single channel recordings in tethered membranes should be possible as it has been shown that a resistance in the G $\Omega$-range can be achieved by reducing membrane diameter below $100 \mu \mathrm{m} .{ }^{30}$ This would only require some moderate methodological improvements, as the membrane architecture we present here would reach an absolute resistance of 30-60 G $\Omega$ if the membrane diameter was reduced from $6 \mathrm{~mm}$ to $0.1 \mathrm{~mm}$.

\section{Conclusion}

We present here the first stable model membrane system in which the insect odorant receptor co-receptor can be incorporate and retain its native structure and function for extended periods of time. The high stability combined with the precise control over experimental conditions afforded by model membrane systems allows the use of a significantly expanded suite of analytical tools to gain detailed insight into protein function that cannot be obtained in another way. By combining electrophysiological techniques with AFM, neutron scattering and others, detailed structural and functional studies become possible, offering unparalleled insight into membrane protein structure and function. With this platform, we show that Orco reacts to significantly lower concentrations of VUAA1 than previously reported when the ligand is introduced via an odorant binding protein rather than an organic solvent. This platform can be used to reconstitute the full OR-Orco complex in order to better understand fundamental behaviour of these receptors as well as screening assays to develop low-impact pesticides. 


\section{References}

(1) Overington, J. P.; Al-Lazikani, B.; Hopkins, A. L. How many drug targets are there? Nature reviews. Drug discovery 2006, 5, 993-996.

(2) Simons, K. Cell membranes: A subjective perspective. Biochimica et biophysica acta 2016, 1858, 2569-2572.

(3) Andersson, J.; Köper, I.; Knoll, W. Tethered Membrane Architectures-Design and Applications. Front. Mater. 2018, 5.

(4) Holt, S. A.; Le Brun, A. P.; Majkrzak, C. F.; McGillivray, D. J.; Heinrich, F.; Lösche, M.; Lakey, J. $\mathrm{H}$. An ion-channel-containing model membrane: structural determination by magnetic contrast neutron reflectometry. Soft matter 2009, 5, 2576-2586.

(5) Kleinschmidt, J. H. Lipid-Protein Interactions 2003; Springer New York: New York, NY, 2019.

(6) Clifton, L. A.; Johnson, C. L.; Solovyova, A. S.; Callow, P.; Weiss, K. L.; Ridley, H.; Le Brun, A. P.; Kinane, C. J.; Webster, J. R. P.; Holt, S. A.; et al. Low resolution structure and dynamics of a colicinreceptor complex determined by neutron scattering. The Journal of biological chemistry 2012, 287, 337346.

(7) Andersson, J.; Köper, I. Tethered and Polymer Supported Bilayer Lipid Membranes: Structure and Function. Membranes 2016, 6, 30.

(8) Vockenroth, I. K.; Ohm, C.; Robertson, J. W. F.; McGillivray, D. J.; Lösche, M.; Köper, I. Stable insulating tethered bilayer lipid membranes. Biointerphases 2008, 3, FA68.

(9) Junghans, A.; Köper, I. Structural analysis of tethered bilayer lipid membranes. Langmuir : the ACS journal of surfaces and colloids 2010, 26, 11035-11040.

(10) Vockenroth, I. K.; Atanasova, P. P.; Jenkins, A. T. A.; Köper, I. Incorporation of alpha-hemolysin in different tethered bilayer lipid membrane architectures. Langmuir 2008, 24, 496-502.

(11) Deplazes, E.; Hartmann, L. M.; Cranfield, C. G.; Garcia, A. Structural Characterization of a CationSelective, Self-Assembled Peptide Pore in Planar Phospholipid Bilayers. The journal of physical chemistry letters 2020, 11, 8152-8156.

(12) Zieleniecki, J. L.; Nagarajan, Y.; Waters, S.; Rongala, J.; Thompson, V.; Hrmova, M.; Köper, I. Cell-Free Synthesis of a Functional Membrane Transporter into a Tethered Bilayer Lipid Membrane. Langmuir: the ACS journal of surfaces and colloids 2016, 32, 2445-2449.

(13) Sutton, T.; Baumann, U.; Hayes, J.; Collins, N. C.; Shi, B.-J.; Schnurbusch, T.; Hay, A.; Mayo, G.; Pallotta, M.; Tester, M.; et al. Boron-toxicity tolerance in barley arising from efflux transporter amplification. Science (New York, N.Y.) 2007, 318, 1446-1449.

(14) Butterwick, J. A.; del Mármol, J.; Kim, K. H.; Kahlson, M. A.; Rogow, J. A.; Walz, T.; Ruta, V. Cryo-EM Structure of the Insect Olfactory Receptor Orco. Nature, 2018, 447-452.

(15) Carraher, C.; Dalziel, J.; Jordan, M. D.; Christie, D. L.; Newcomb, R. D.; Kralicek, A. V. Towards an understanding of the structural basis for insect olfaction by odorant receptors. Insect Biochemistry and Molecular Biology 2015, 66, 31-41.

(16) Jones, P. L.; Pask, G. M.; Rinker, D. C.; Zwiebel, L. J. Functional agonism of insect odorant receptor ion channels. Proceedings of the National Academy of Sciences of the United States of America 2011, 108, $8821-8825$.

(17) Hopf, T. A.; Morinaga, S.; Ihara, S.; Touhara, K.; Marks, D. S.; Benton, R. Amino acid coevolution reveals three-dimensional structure and functional domains of insect odorant receptors. Nature communications 2015, 6, 6077.

(18) Yan, H.; Opachaloemphan, C.; Mancini, G.; Yang, H.; Gallitto, M.; Mlejnek, J.; Leibholz, A.; Haight, K.; Ghaninia, M.; Huo, L.; et al. An Engineered orco Mutation Produces Aberrant Social Behavior and Defective Neural Development in Ants. Cell 2017, 170, 736-747.e9. 
(19) Trible, W.; Olivos-Cisneros, L.; McKenzie, S. K.; Saragosti, J.; Chang, N.-C.; Matthews, B. J.; Oxley, P. R.; Kronauer, D. J. C. orco Mutagenesis Causes Loss of Antennal Lobe Glomeruli and Impaired Social Behavior in Ants. Cell 2017, 170, 727-735.e10.

(20) Chakrabarti, P.; Rana, S.; Bandopadhyay, S.; Naik, D. G.; Sarkar, S.; Basu, P. Field populations of native Indian honey bees from pesticide intensive agricultural landscape show signs of impaired olfaction. Sci Rep 2015, 5, 12504.

(21) Lusebrink, I.; Girling, R. D.; Farthing, E.; Newman, T. A.; Jackson, C. W.; Poppy, G. M. The Effects of Diesel Exhaust Pollution on Floral Volatiles and the Consequences for Honey Bee Olfaction. Journal of chemical ecology 2015, 41, 904-912.

(22) Eggleton, P. The State of the World's Insects. Annu. Rev. Environ. Resour. 2020, 45, 61-82.

(23) Reddy, G. V. P.; Guerrero, A. Interactions of insect pheromones and plant semiochemicals. Trends in Plant Science 2004, 9, 253-261.

(24) Cook, S. M.; Khan, Z. R.; Pickett, J. A. The use of push-pull strategies in integrated pest management. Annu. Rev. Entomol. 2007, 52, 375-400.

(25) Colm Carraher; Ali Reza Nazmi; Richard D. Newcomb; Andrew Kralicek. Recombinant expression, detergent solubilisation and purification of insect odorant receptor subunits. Protein Expression and Purification 2013, 90, 160-169.

(26) Mukunda, L.; Lavista-Llanos, S.; Hansson, B. S.; Wicher, D. Dimerisation of the Drosophila odorant coreceptor Orco. Frontiers in Cellular Neuroscience 2014, 8, 261.

(27) Murugathas, T.; Zheng, H. Y.; Colbert, D.; Kralicek, A. V.; Carraher, C.; Plank, N. O. V. Biosensing with Insect Odorant Receptor Nanodiscs and Carbon Nanotube Field-Effect Transistors. ACS applied materials \& interfaces 2019, 11, 9530-9538.

(28) Yamada, T.; Sugiura, H.; Mimura, H.; Kamiya, K.; Osaki, T.; Takeuchi, S. Highly sensitive VOC detectors using insect olfactory receptors reconstituted into lipid bilayers. Sci. Adv. 2021, 7, eabd2013.

(29) Schiller, S. M.; Naumann, R.; Lovejoy, K.; Kunz, H.; Knoll, W. Archaea analogue thiolipids for tethered bilayer lipid membranes on ultrasmooth gold surfaces. Angew. Chem. Int. Ed. 2003, 42, 208-211.

(30) Vockenroth, I. K.; Fine, D.; Dodobalapur, A.; Jenkins, A. T. A.; Köper, I. Tethered bilayer lipid membranes with giga-ohm resistances. Electrochemistry Communications 2008, 10, 323-328.

(31) Vogel, N.; Zieleniecki, J.; Köper, I. As flat as it gets: ultrasmooth surfaces from template-stripping procedures. Nanoscale 2012, 4, 3820-3832.

(32) Andersson, J.; Knobloch, J. J.; Perkins, M. V.; Holt, S. A.; Köper, I. Synthesis and Characterization of Novel Anchorlipids for Tethered Bilayer Lipid Membranes. Langmuir : the ACS journal of surfaces and colloids 2017, 33, 4444-4451.

(33) Knoll, W.; Frank, C.; Heibel, C.; Naumann, R.; Offenhäusser, A.; Rühe, J.; Schmidt, E.; Shen, W.; Sinner, A. Functional tethered lipid bilayers. Reviews in Molecular Biotechnology 2000, 74, 137-158.

(34) Vockenroth, I. K.; Rossi, C.; Shah, M. R.; Köper, I. Formation of tethered bilayer lipid membranes probed by various surface sensitive techniques. Biointerphases 2009, 4, 19-26.

(35) Sleytr, U. B.; Schuster, B.; Pum, D. Nanotechnology and biomimetics with 2-D protein crystals. IEEE engineering in medicine and biology magazine : the quarterly magazine of the Engineering in Medicine \& Biology Society 2003, 22, 140-150.

(36) Wang, B.; Liu, Y.; Wang, G.-R. Chemosensory genes in the antennal transcriptome of two syrphid species, Episyrphus balteatus and Eupeodes corollae (Diptera: Syrphidae). BMC Genomics 2017, 18, 586. (37) Andersson, J.; Fuller, M. A.; Wood, K.; Holt, S. A.; Köper, I. A tethered bilayer lipid membrane that mimics microbial membranes. Phys. Chem. Chem. Phys. 2018, 20, 12958-12969.

(38) Spinelli, S.; Lagarde, A.; Iovinella, I.; Legrand, P.; Tegoni, M.; Pelosi, P.; Cambillau, C. Crystal structure of Apis mellifera OBP14, a C-minus odorant-binding protein, and its complexes with odorant molecules. Insect Biochemistry and Molecular Biology 2012, 42, 41-50. 
(39) Notman, R.; Noro, M.; O'Malley, B.; Anwar, J. Molecular basis for dimethylsulfoxide (DMSO) action on lipid membranes. Journal of the American Chemical Society 2006, 128, 13982-13983.

(40) Sisi Chen; Luetje, C. W. Inhibition of odorant and Orco agonist initiated current responses of oocytes expressing insect odorant receptors by various amines, 2014. 